\title{
A comunicação do diagnóstico "sombrio" na relação médico-paciente entre estudantes de Medicina: uma experiência de dramatização na educação médica
}

\author{
Communicating an unfavorable diagnosis during \\ the physician-patient relationship: a role-playing \\ experiment with medical students
}

\author{
Natalia Braga Hortêncio Jucá ${ }^{1}$ \\ Annatália Meneses de Amorim Gomes ${ }^{\mathrm{II}}$ \\ Lucas Silvestre Mendes ${ }^{\mathrm{I}}$ \\ Diego Morais Gomes ${ }^{\mathrm{I}}$ \\ Bruna Vitória Lima Martins ${ }^{\mathrm{I}}$ \\ Carlos Maximiliano Gaspar Carvalho Silva ${ }^{\mathrm{I}}$ \\ Carolina Arcanjo Lino ${ }^{\mathrm{I}}$ \\ Kathiane Lustosa Augusto ${ }^{\mathrm{I}}$ \\ Andrea Caprara ${ }^{\mathrm{I}}$
}

PALAVRAS-CHAVE

- Relações Médico-Pacient

- Estudantes de Medicina

- Educação Médica

\section{KEY WORDS}

- Role Playing

- Psychodrama

- Physician-Patient Relations

- Students, Medical

- Education, Medical

Recebido em: 28/06/2008 Reencaminhado em: 17/03/2009 Aprovado em: 18/06/2009

REVISTA BRASILEIRA DE EDUCAÇ̃̃O MÉDICA $34(1): 57-64: 2010$

\begin{abstract}
R E S U M O
O objetivo do trabalho foi apresentar a dramatização como prática para a humanização da relação médico-paciente entre estudantes do terceiro semestre do curso de Medicina da Universidade Estadual do Ceará (Uece). Por meio de metodologia qualitativa, aplicaram-se perguntas referentes aos sentimentos vivenciados enquanto na posição de médicos e de pacientes, e à vivência com a técnica, após a mesma ser apresentada em sala de aula $(n=24)$. Os dados permitiram identificar que os temas abordados se referiam a doenças graves e perdas. Em relação aos sentimentos, no papel tanto de paciente quanto de médico, demonstraram angústia, impotência, apreensão, medo e ansiedade. Expressaram dificuldades frente ao diagnóstico e à transmissão de má notícia, ao mesmo tempo em que valorizaram a construção do vínculo médico-paciente. Frente à experiência, destacaram a técnica como geradora de autoconhecimento e vivência da futura profissão. Estudos prospectivos devem ser realizados para avaliar o impacto dessa estratégia na humanização da relação médico-paciente e educação médica, explorando o máximo de potencialidade frente às suas limitações.
\end{abstract}

\section{A B S T R A C T}

The aim of this article is to present role-playing as a practice for humanization of the physician-patient relationship, as experienced by third-semester medical students at the State University of Ceará (UECE), Brazil. Using a qualitative methodology, questions were applied relating to the students' feelings while playing the roles of doctor and patient, respectively, and their experience with the technique after its presentation in the classroom $(n=24)$. According to the findings, the topics that were approached related to serious illnesses and losses. Both in the patient's and physician's role, the students felt anguish, powerlessness, apprehension, fear, and anxiety. They expressed difficulties towards both the diagnosis itself and communicating the bad news, even while they valued building and consolidating the physician-patient bond. As for the method, they highlighted its importance for generating self-knowledge and experience for their future profession. Prospective studies should be performed to evaluate the strategy's impact on the humanization of the physician-patient relationship and medical education, fully exploring its potential and limitations.

\footnotetext{
Universidade Estadual do Ceará, Fortaleza, CE, Brasil.

${ }^{I I}$ Universidade Estadual do Ceará, Fortaleza, CE, Brasil; Secretaria de Saúde do Estado do Ceará. Coordenadoria de Apoio ao Desenvolvimento da Atenção em Saúde, Fortaleza, CE, Brasil.
} 


\section{INTRODUÇÃO}

A relação médico-paciente constitui um desafio no contexto atual da medicina no tocante à humanização do encontro clínico. Há uma assimetria nesta relação que é parte de uma complexa rede de representações conflituosas, disparidades de classe, status, escolaridade e condições de vida entre médicos e seus pacientes ${ }^{1-4}$.

É importante considerar que esse conflito não se deve somente aos fatores provenientes dos próprios sujeitos, mas está permeado por questões macroestruturais. A prática clínica é atravessada por uma precária estrutura, gestão elitista e centralizadora, difícil acesso, desorganização e tecnicismo ${ }^{5}$.

Além disso, a formação acadêmica não prepara o profissional para escutar a experiência do paciente, o que compromete a qualidade do cuidado $\mathrm{o}^{6,7}$. O formato do sistema tradicional de ensino retarda o contato com o paciente, que somente ocorrerá nos últimos anos do curso com a vivência intensiva do internato. A delonga na prática da relação com o paciente leva o acadêmico a deparar-se com situações-limite consigo mesmo, com seu paciente e com a própria ciência médica ${ }^{8}$.

É reconhecida a dificuldade dos profissionais de saúde em lidar com a dimensão subjetiva de sua prática. O Ministério da Saúde criou em 2003 a Política Nacional de Humanização, que visa ao fortalecimento dos princípios do Sistema Único de Saúde (SUS), como a universalidade, integralidade e equidade ${ }^{9,10}$. Esta surge como possibilidade de melhoria do contato humano dos profissionais com os usuários do sistema e prevê a transformação do modelo de atenção vigente, baseado na doença e na queixa-conduta, num modelo centrado no usuário e holístico, aliado à clínica ampliada, ao vínculo e à corresponsabilidade por meio de uma gestão democrática ${ }^{11}$. Maior equidade na relação de cunho terapêutico exige do profissional a competência para cuidar, aliada à autonomia do paciente ${ }^{12}$.

Nas últimas décadas, estudos sobre métodos de ensino-aprendizagem mais apropriados à questão da humanização na relação médico-paciente vêm sendo desenvolvidos com estudantes e profissionais da saúde ${ }^{13-18}$. Todas essas estratégias educacionais convergem para alguns elementos comuns: o uso de metodologias ativas, centradas nos participantes, e o trabalho em pequenos grupos, em busca de reflexão e desenvolvimento de competências para lidar com os diferentes tipos de pacientes e de personalidades ${ }^{19}$.

No contexto destas metodologias, destaca-se o psicodrama. Formulado no início do século 20 por Jacob Levy Moreno, propõe, por meio da espontaneidade e da criatividade, recompor experiências vivenciadas por um sujeito ou grupo, criando novos conceitos para o desenvolvimento de diferentes papéis sociais ${ }^{20,21}$.
O psicodrama mostrou-se também, ao longo do tempo, um interessante meio de apoio aos educadores, nos mais diversos campos de atuação, fornecendo um conjunto de estratégias valiosas ao processo de ensino, tais como o teatro espontâneo, o jornal vivo, os jogos dramáticos, o sociodrama e a dramatização ${ }^{21,22}$. Entre seus desdobramentos pedagógicos, destaca-se o método educacional psicodramático ou psicodrama pedagógico, desenvolvido por Romaña ${ }^{23}$. Este método pode ser utilizado em situações especiais, em que o conhecimento decorre de uma aprendizagem simultânea à experiência de vida. A vivência do dia a dia é vista em todas as suas nuances, facilitando a compreensão da didática interpessoal.

Nessa dinâmica das relações humanas, a dramatização é um recurso psicodramático que permite a troca de papéis. Baseado na ideia do "como se", permite que a pessoa "atue" angústias, medos e ansiedades presentes em seu papel profissional, incluindo todos os aspectos que sua possibilidade criativa lhe permita, no intuito de compreender as tensões provocadas no exercício deste papel. Sua finalidade é, de maneira espontânea, proporcionar a experimentação dos pontos de vista de outras pessoas, ao interpretar o papel de outrem ${ }^{24}$. Aplicado ao campo da educação médica, visa à percepção objetiva de sentimentos e atitudes frente à prática profissional, sendo utilizado com estudantes e profissionais de diversas especialidades médicas ${ }^{25-27}$.

Motivados pelo desafio de incluir na formação médica elementos que contribuam para humanizar a relação médico-paciente, interrogamos: até que ponto a dramatização aumenta a consciência e a sensibilidade dos acadêmicos quanto a aspectos importantes da relação médico-paciente? Quais os principais sentimentos apresentados por aqueles que dela participam ao experimentarem os papéis de médico e de paciente nos anos iniciais da formação?

O objetivo deste trabalho é apresentar e discutir o método da dramatização, suas aplicações e limitações como ferramenta na formação do estudante em aspectos da relação médico-paciente.

\section{METODOLOGIA}

As atividades descritas foram desenvolvidas ao longo da disciplina Ciências Sociais e Saúde, composta de quatro créditos, em outubro e novembro de 2007, ministrada a 40 acadêmicos do terceiro semestre.

Na disciplina, abordaram-se as capacidades de comunicação, especialmente a de escuta, empregando-se a técnica da dramatização como ferramenta de ensino-aprendizagem. O papel de paciente desenvolve nos alunos um interesse pela experiência subjetiva da doença, permitindo explorar melhor os fatores que interferem 
no cuidado do paciente ${ }^{28}$. Aqueles que experimentam o papel de médico desenvolvemramenta de ensino-aprendizagem.s de pacientes e de personalidades a escuta ativa, tentando entender o que o paciente sente e como interpreta a própria doença, além de valorizarem o que ele está dizendo.

A dinâmica foi dividida em cinco momentos:

1. integração dos participantes e aquecimento para a vivência em grupo: buscou colocar os alunos, a maioria pela primeira vez, em contato com a experiência psicodramática; os facilitadores fizeram uma breve exposição sobre o conceito de dramatização, a atividade e os papéis de protagonistas (médico e paciente) e observador, os objetivos e o tempo de cada dramatização;

2. formação espontânea de trios para os papéis de médico, paciente e observador;

3. dramatização: os trios escolheram os temas livremente; cada aluno teve a oportunidade de experimentar as três possibilidades de papéis propostos (médico, paciente e observador); quando observador, o aluno foi orientado a anotar os pontos mais relevantes que percebeu na relação médico-paciente vivenciada pelos demais protagonistas do trio;

4. compartilhamento e comentários sobre a vivência: os trios retornam à formação inicial de grande roda, e cada indivíduo tem a possibilidade de interagir com o grupo, expressando suas experiências, emoções e dificuldades frente ao processo educacional;

5. finalização: realizada pelos facilitadores da atividade; houve leitura de questões emergentes, destacando-se pontos-chave sobre as percepções dos papéis dramatizados e os vínculos vivenciados em cena, acrescentando-se observações consideradas relevantes.

Ao fim da dinâmica, os alunos foram orientados a responder a três questões abertas: Como se sentiu/percebeu no papel de paciente?; Como se sentiu/percebeu no papel de médico?; Discorra livremente sobre a vivência com a dramatização.

A participação foi voluntária, com a garantia de anonimato dos participantes. Os nomes utilizados para identificar os entrevistados são fictícios.

Os cuidados com os preceitos éticos foram tomados conforme a resolução da Portaria 196/96, que envolve pesquisas com seres humanos ${ }^{29}$. O projeto foi submetido ao Comitê de Ética da Uece, sendo aprovado sob o protocolo $\mathrm{n}^{\mathrm{o}}$ 08.185.581-8.

\section{RESULTADOS E DISCUSSÃO}

Do total de 40 alunos, 24 se dispuseram a participar da atividade, entre eles 18 homens e 6 mulheres, com idades entre 18 e
27 anos. As respostas obtidas deram origem a quatro categorias: temas abordados; sentimentos vivenciados nos papéis de paciente e de médico; dificuldades frente ao diagnóstico; vivência do método.

\section{Temas abordados}

As temáticas escolhidas pelos estudantes para a dramatização estavam relacionadas a doenças (leucemia, HIV-Aids) ou perdas (amputação de membro por complicação diabética, perda fetal). De modo geral, observou-se que os temas encenados refletem uma visão paradigmática do modelo biomédico da medicina centrada na doença ${ }^{30,31}$. Esses temas parecem suscitar, nesse período inicial do curso, medos e fantasias a respeito do que é ser médico, influenciando as escolhas.

\section{Sentimentos vivenciados nos papéis de paciente e de médico}

Os estudantes, no papel de pacientes, revelaram sentimentos de angústia, impotência, apreensão, medo, tristeza e ansiedade: "Senti-me angustiado ao saber que não havia nada a se fazer [...]" (João, 19 anos). "Eu senti muito medo no momento da revelação do diagnóstico [...]" (Patrick, 18 anos).

Essas emoções são congruentes com a fase inicial de estudos em que se encontram os alunos. Neste momento, a mobilização e a expressão de sentimentos são maiores, uma vez que eles ainda não desenvolveram formas de atuação profissional, adquiridas em anos posteriores na formação. A condição de estudante ainda permite admitir mais facilmente fragilidades, limitações, receios.

No papel de médicos, os alunos expuseram a mesma gama de sensações vivenciadas no lugar de pacientes, acrescentando-se, ainda, o despreparo e a dificuldade de comunicação: "Senti-me impotente diante da situação [...] Inseguro acerca da aceitação do paciente e das implicações da notícia no prognóstico da sua vida" (Filipe, 22 anos). "Não me senti nem um pouco à vontade para dar a notícia [perda fetal] [...]. Não sabia como lidar com a situação, que palavras usar, como confortar a mulher desamparada à minha frente" (Maria, 19 anos).

Esse posicionamento do estudante na figura de médico pode ser compreendido como decorrente do fardo de responsabilidade. A ansiedade e o medo de uma avaliação negativa pelo paciente podem fazer com que o médico relute em transmitir o diagnóstico e, como nos casos citados, um mau prognóstico ${ }^{32,33}$.

\section{Comunicação de diagnóstico "sombrio"}

A experiência permitiu desenvolver a temática da comunicação de más notícias numa consulta clínica, revelando que esta é uma importante habilidade a ser desenvolvida no futuro doutor: “Nessa brincadeira eu percebi algo que nem imaginava: o quan- 
to sou despreparada para abordar esse tema [o de comunicar o diagnóstico de câncer]" (Eduarda, 25 anos).

Na tentativa de comunicação destes diagnósticos, observou-se excesso de rodeios do médico, excessiva subjetividade e uso de linguagem coloquial não habitual no nível cultural do paciente, provocando insegurança e ansiedade neste, como observado nas falas de alguns entrevistados: “[...] E o excesso de cuidado do médico aumentou minha preocupação porque ficava mais claro que se tratava de algo grave" (Maria, 19 anos). "[...] Parecia que até ele [o médico] tinha desconfiança sobre o meu problema. Ficava indagando, sem muita objetividade, para depois dizer que tinha um problema grave. Senti-me quase morto" (Alessandra, 22 anos).

Por outro lado, também se observou excesso de objetividade do médico durante a consulta, tornando agressiva a comunicação do diagnóstico e ansioso o paciente: “[...] O médico, ao fazer o diagnóstico, relatou-me de forma muito direta e, de certa forma, agressiva, fazendo-me questionar se eu ainda queria saber mais sobre minha doença" (Cleriston, 27 anos).

O equilíbrio, nem sempre fácil, entre as forças da objetividade e da sutileza da informação, sem transparecer receio ou hesitação em seu relato, também foi observado nas dramatizações: “ Não prometi cura, mas expliquei como era o tratamento e disse que a manteria informada da evolução da doença. [...] A minha maneira de tranquilizar o paciente foi ser sempre sincero com o mesmo" (Patrick, 18 anos). "É difícil tranquilizar o paciente inteiramente, mas, quando se coloca à disposição deste a confiança de uma terapêutica segura e adequada, o paciente consegue acreditar e aderir ao tratamento" (Victor, 21 anos).

Pelos depoimentos, a desafiadora arte de comunicar uma notícia ruim ao paciente fica bastante evidente. Há um bloqueio tanto por parte do médico em exercer essa tarefa, evitando uma situação que poderia fazê-lo sentir-se impotente e fracassado, quanto por parte do paciente, que interpreta o fato como uma sentença de morte. Os sentimentos e anseios presentes nas duas faces da relação podem prejudicar a boa comunicação: “O médico fica com medo de dar a informação de forma súbita e rude, e, ao mesmo tempo, fica com receio de dar uma informação muito otimista, que fuja da realidade" (Clara, 19 anos).

Tapajós $^{34}$ reconhece em seus estudos a existência de uma atitude de desqualificação em situações de difícil manuseio emocional, a qual implica comunicar-se de maneira a invalidar as comunicações própria e alheia:

[...] declarações contraditórias, frases incompletas, interpretações espúrias, estilo obscuro, ou seja, a arte sutil de nada comunicar enquanto se diz algo. Ela siste- maticamente opera na comunicação de pessoas que querem rapidamente evadir-se de situações que, de alguma forma, soam difíceis, quais sejam, aquelas nas quais essas pessoas preferem não comunicar, de modo a esquivar-se do compromisso inerente a qualquer tipo de comunicação (p. 167).

No intuito de minimizar essas dificuldades, Buckman publicou, no início da década de 1990, o protocolo Spikes, hoje uma das referências metodológicas mais adotadas internacionalmente no que se refere à comunicação de más notícias ${ }^{35}$. Na maioria das vezes, o médico não foi devidamente habilitado a gerenciar tais situações durante sua formação acadêmica e precisa aprender na prática a desenvolver habilidades relacionais e comunicacionais a fim de obter melhor conhecimento da personalidade do paciente: "[...] Com o decorrer da conversa, dá para avaliar o grau de entendimento, bem como os possíveis medos do cliente em relação a sua enfermidade. Após essa análise, dá pra decidir a melhor forma de comunicar a situação" (Tiago, 26 anos).

Quando na situação de pacientes, a grande maioria dos alunos deixou explícita a vontade de serem informados sobre seus diagnósticos, prognósticos e terapêuticas, como mostra o relato a seguir:

Inicialmente, o médico procurou saber meu estado,
como os meus sintomas estavam progredindo e se eu
queria, de fato, saber meu diagnóstico. Optei por co-
nhecê-lo, quis saber se tinha cura, se era rápido, quan-
do poderia começar o tratamento. O médico informou
o significado da doença e como ocorreria o tratamen-
to. (Paulo Pedro, 25 anos)

Um único sujeito observou a recusa em receber uma má notícia. Deve-se ressaltar, contudo, a superioridade e o respeito à vontade do paciente de saber ou não seu diagnóstico. Trata-se do direito legítimo à informação, que precisa ser garantido na relação de cuidado, ao permitir o protagonismo do paciente nas decisões sobre conduta e prognóstico: “[...] eu acho que o paciente não deveria receber a má notícia, a menos que o paciente insistisse muito. [...] É sempre muito ruim para o paciente saber que resta pouco tempo de vida" (Rodrigo, 24 anos).

As impressões acima corroboram os estudos de Kaufmann ${ }^{36}$, para quem a dramatização permite ao estudante jogar com as diversas possibilidades criativas de desempenho do papel, para então refletir sobre as formas adequadas de abordar e comunicar diagnósticos em situações inoportunas frente às mais diversas situações. 


\section{Perspectiva dos estudantes sobre a vivência da dramatização}

Para a maioria dos estudantes, a resposta foi positiva frente ao método. Entre as opiniões formuladas, a dramatização foi vista como um bom exercício de simulação da realidade, agente aguçador de sentimentos, possibilitadora de vivência de situações do cotidiano, treino de papéis para a futura profissão, facilitadora de competências.

Aspecto muito destacado pelos alunos foi a utilização desse recurso psicodramático como forma de autoconhecimento por meio da prática dos papéis, como observado nos seguintes trechos: "Quando refletimos de forma aplicada, conseguimos aguçar sentimentos que já experimentamos" (João, 19 anos). “[...] Experiência válida no sentido de nos fazer perceber a angústia do médico em dar uma má notícia, ver como é difícil [...]" (Roberto, 24 anos).

Outros alunos realçaram a importância da troca de papéis e, principalmente, a obtenção de diferentes pontos de vista como contribuintes ao aprendizado e à compreensão da essência da relação médico-paciente. Essas ações corroboram a proposta de Moreno $^{21}$, que define a inversão de papéis como a possibilidade de desenvolver atitudes como empatia e solidariedade, aprendendo a se colocar no lugar do outro: "Achei uma prática interessante, pois pode-se ver todos os pontos de vista [...]" (Antônio, 21 anos). "O jogo de papéis foi construtivo, pois permitiu analisar diferentes pontos de vista, principalmente do paciente [...]" (Gustavo, 24 anos).

A grande maioria dos estudantes destacou o papel da dramatização em preparar os futuros médicos para o contato com os clientes, diminuir a ansiedade dos alunos no contato com o doente e lidar com o papel de onipotente atribuído ao médico, o que se evidencia nos seguintes depoimentos: "[...] permitindo que possamos encarar problemas do cotidiano e aprendermos como superá-los" (Noemi, 20 anos). "Fomos colocados em situações que, provavelmente, viveremos durante o exercício da nossa profissão. Assim, tenho uma noção de como agir e do que esperar em situações difíceis" (Ronnie, 20 anos).

Segundo Kaufman ${ }^{36}$, um dos principais objetivos da dramatização, no contexto da saúde e da relação médico-paciente, é desenvolver capacidades no sentido de promover um atendimento médico humanizado em que o paciente não seja visto como um simples número ou doença: "[...] para que possamos aprimorar nossas técnicas na relação médico-paciente" (José, 23 anos). "Facilita o processo de abordagem do profissional de saúde diante da difícil situação do paciente [...]" (Tiago, 26 anos).
É importante para os alunos mostrar a dramatização não como uma prática que vise caracterizar suas atitudes como certas ou erradas, mas, sim, como uma ação formadora de opinião e senso crítico $^{37}$. $\mathrm{O}$ autoconhecimento proporcionado pela atividade permite ao aluno desmistificar a onipotência do médico, fazendo-o perceber suas limitações e estimulando-o a aprimorar continuamente suas habilidades: "[o exercício] foi muito importante porque despertou a atenção para a falta de preparo de todos nós para darmos uma má notícia. [...] Que um dia eu não chegue diante de um caso desses sem saber como me portar" (Eduarda, 25 anos).

\section{Limitações do método}

Adramatização tem sido um pilar na avaliação de comportamentos durante décadas ${ }^{38}$. Nos últimos anos, sua aplicabilidade foi estendida aos estudos sociais na área médica, dentro da qual ainda se observam algumas limitações práticas.

Primeiramente, o método pode suscitar variados graus de ansiedade entre os membros participantes, o que limita o sentimento de entrega dos mesmos diante da atividade - ansiedade esta frente à possibilidade de conflitos de papéis, à inibição pelo uso da expressão corporal e à preocupação demasiada em corresponder às expectativas do grupo. Aideia da representação teatral se demonstraria desvinculada das emoções individuais ${ }^{39}$, como demonstrado nos trechos a seguir: "[...] não ter a pressão psicológica da situação real" (André, 18 anos). "[...] não consegue tocar nos meus sentimentos, possivelmente afetando a simulação" (Maria, 19 anos).

Além disso, o método exige maior compromisso e participação dos executores frente ao processo de aprendizagem, o que pode se confrontar com a inexperiência deles. Haveria uma preocupação demasiada em satisfazer os anseios do gerenciador da atividade, em detrimento dos sentimentos expressos pelos demais participantes ou mesmo das crenças individuais. Como retrata Zinker ${ }^{40}$, nesses casos, o sistema seria como uma roda de carroça quebrada, devido à interligação fraca que envolve os membros do grupo e à comunicação vicária com a ação do centro (terapeuta): “A roda quebrada não gira; as pessoas experienciam um contato terapêutico com o terapeuta e a pessoa na 'cadeira vazia', porém têm pouca afinidade entre si, além da ansiedade gerada por ocuparem o centro do grupo [...]"(p. 233).

Além disso, os relatos disponíveis até o momento são geralmente com grupos pequenos, realizados em espaços de tempo restritos, mostrando-se incapazes de gerar senso crítico e modificar pensamentos e retóricas estabelecidos. Nos estudos de Thom ${ }^{41}$, uma amostra randomizada de 20 médicos (10 no grupo 
controle, 10 no grupo de intervenção) foi estudada quanto à opinião dos pacientes frente à confiança na relação, à satisfação no atendimento e à autoadesão ao tratamento. A amostra de intervenção foi exposta a dinâmicas psicodramáticas e discussões em grupo sobre experiências e melhoria de atendimento. Após quatro sessões, os grupos foram confrontados, e, na visão dos pacientes, não houve diferenças significativas na prática. Este ponto de vista também é defendido por outros autores ${ }^{42-44}$.

\section{CONSIDERAÇÕES FINAIS}

As novas diretrizes curriculares para o ensino médico no País têm estimulado as atividades práticas desde os primeiros anos do curso, em especial as que promovam um olhar mais humanizado e mais próximo das necessidades das populações. É de interesse que essas ações propiciem, de igual modo, mudanças em relação aos sentimentos e atitudes vivenciados pelos alunos.

Uma avaliação longitudinal e prospectiva deverá ser conduzida para mensurar o impacto dessas mudanças no processo formativo. Uma atividade isolada em meio aos seis anos de curso não representa uma força transformadora sobre a segurança do profissional frente a situações difíceis, a relações médico-paciente complexas, nem à adoção de habilidades de comunicação e atitudes humanizadas.

A procura por estratégias que possibilitem a melhoria do contato humano entre médico e paciente estimula, assim, a criação e a sustentação de espaços de comunicação permanentes que favorecem a livre expressão, o respeito à diversidade de opiniões e a criatividade. Nesse sentido, o psicodrama é uma das ferramentas cabíveis, porque possibilita a expressão de afetos comuns ao conjunto de alunos, sem expor a fragilidade de cada um individualmente.

O estudo confirma o pressuposto de que a verbalização de angústias, ansiedades e medos, corroborada pela expressão cênica destes, possibilita elaborar melhor tais sentimentos e enriquece a formação humanística, crítica e participativa dos indivíduos frente à realidade social que vivenciarão na prática clínica.

São necessários outros estudos nesta área, em diferentes contextos, com diferentes sujeitos, para que se possa verificar de fato o real alcance da estratégia, explorando o máximo de potencialidade frente às suas limitações.

\section{REFERÊNCIAS}

1. Caprara A, Rodrigues J. A relação assimétrica médico-paciente: repensando o vínculo terapêutico. Ciên Saúde Colet. 2004;9:139-46.
2. Ribeiro MMF, Amaral CFS. Medicina centrada no paciente e ensino médico: a importância do cuidado com a pessoa e o poder médico. Rev Bras Educ Med. 2008;32(1):90-7.

3. Sucupira AC. A importância do ensino da relação médico-paciente e das habilidades de comunicação na formação do profissional de saúde. Interface Comun Saúde Educ. 2007;11(23):624-7.

4. Coelho Filho JM. Relação médico-paciente: a essência perdida. Interface Comun Saúde Educ. 2007;11(23):631-33.

5. Bosi MLM, Affonso KC. Direito à saúde e participação popular: confrontando as perspectivas de profissionais e usuários da rede pública de serviços de saúde. In: Bosi MLM, Mercado-Martínez FJ, orgs. Pesquisa qualitativa de serviços de saúde. Petrópolis: Vozes; 2004. p. 451-79.

6. Caprara A. Uma abordagem hermenêutica da relação saúde-doença. Cad Saúde Pública. 2003;19(4):923-31.

7. Ceccim RB. Educação permanente em saúde: desafio ambicioso e necessário Interface Comun Saúde Educ. 2005;9(16):161-8.

8. Ramos-Cerqueira ATA, Lima MCP, Reis JRT, Fonseca NMV. Era uma vez... contos de fadas e psicodrama auxiliando alunos na conclusão do curso médico. Interface Comun Saúde Educ. 2005;9(16):81-9.

9. Brasil. Ministério da Saúde. Secretaria Executiva. Núcleo Técnico da Política Nacional de Humanização. HUMANIZASUS: Política Nacional de Humanização. Documento-base. Brasília: Ministério da Saúde; 2006.

10. Benevides R, Passos E. A humanização como dimensão política das políticas de saúde. Ciên Saúde Colet. 2005;10(3):561-71.

11. Campos GWS. Humanização na saúde: um projeto em defesa da vida?. Interface Comun Saúde Educ. 2005; 9:389-406.

12. Merhy EE. Saúde: a cartografia do trabalho vivo. São Paulo: Hucitec; 2002.

13. Freire P. Pedagogia da autonomia: saberes necessários à prática educativa. São Paulo: Paz e Terra; 2001.

14. Freire P. Pedagogia do oprimido. São Paulo: Martins Fontes; 2006.

15. Kolb DA. Experimental Learning: experience as the source of learning and development. New Jersey: Englewood Cliffs; 1984.

16. Barrows HS. How to design a problem-based curriculum for the preclinical years. New York: Springer Pub Co; 1985.

17. Mamede S, Penaforte J. Aprendizagem baseada em problemas. São Paulo: Hucitec; 2001. 
18. Cyrino EG, Torralles-Pereira ML. Discovery-based teaching and learning strategies in health: problematization and problem-based learning. Cad Saúde Pública. 2004;20(3):780-8.

19. Caprara A. Escuta como cuidado: é possível ensinar?. In: Pinheiro R, Matos RA, orgs. Razões públicas para a integralidade em saúde: o cuidado como valor. Rio de Janeiro: Cepesc; Abrasco; 2007. p. 240-2.

20. Merhy EE, Onoko R. Agir em Saúde: um desafio para o público. São Paulo: Hucitec; 2002.

21. Moreno JL. Psicodrama. São Paulo: Cultrix; 1975.

22. Moreno JL. Quem sobreviverá? Fundamentos da sociometria, psicoterapia de grupo e psicodrama. São Paulo: Dimensão; 1974. v. 1

23. Romaña MA. Psicodrama pedagógico: método educacional psicodramático. Campinas: Papirus; 1987.

24. Garrido Martin E. Psicologia do Encontro. São Paulo: Duas Cidades; 1996.

25. Kaufman A. Teatro Pedagógico: bastidores da iniciação médica. São Paulo: Agora; 1992.

26. Perazzo S. Reflexões de um psicodramatista: o diretor, seu papel e sua integração aos objetivos pedagógicos do grupo de role-playing. Rev FEBRAP. 1980; 3(1):280-6.

27. Cuschini L. Uma experiência de orientação de médicos residentes em psiquiatria com a técnica do role-playing. Rev FEBRAP. 1978;1(2):91.

28. Luz MT. Fragilidade social e busca de cuidado na sociedade civil de hoje. In: Pinheiro R; Mattos RA, orgs. Cuidado: as fronteiras da integralidade. Rio de Janeiro: Hucitec; 2004. p. 9-20.

29. Brasil. Ministério da Saúde. Resolução n. ${ }^{\circ}$ 196, de 10 de outubro de 1996. Dispõe sobre as diretrizes e a regulamentação de pesquisas envolvendo seres humanos. Diário Oficial da União, Brasília, 16 out. 1996; Seção 1, p.15-25.

30. Fontes BASM, Lacerda de Melo NM, Leal SMR, Fuentes EP. Gestão local no Nordeste do Brasil: em busca de novos paradigmas. Rev Mexicana Sociología 1995; 57(2):123-42.

31. Nations MK, Gomes AMA. Cuidado. "cavalo batizado" e crítica da conduta profissional pelo paciente-cidadão hospitalizado no Nordeste brasileiro. Cad Saúde Pública. 2007;23(9):2103-12.

32. Vianna A, Piccelli H. O estudante, o médico e o professor de medicina perante a morte e o paciente terminal. Rev Assoc Med Bras. 1998;44(1):21-7.

33. Van der Molen HT. Habilidades de transmissão de más notícias. In: Madeiro Leite AJ, Caprara A, Coelho Filho JM, orgs. Habilidades de comunicação com pacientes e famílias. São Paulo: Sarvier; 2007. p.78-88.

34. Tapajós R. Breaking bad news and the pragmatics of human communication: the use of cinema in teaching/learning activities in medical education. Interface Comun Saúde Educ. 2007; 11(21):165-72.

35. Buckman R. Breaking bad news: a guide for health care professionals. Baltimore: John Hopkins University Press; 1992.

36. Kaufman A. Role-playing. In: Monteiro RF, org. Técnicas fundamentais do Psicodrama. São Paulo: Agora; 1998. p.151-61.

37. Kleinman A. The ilness narratives: suffering, healing \& the human condition. New York: Basic Book; 1988.

38. Van Hasselt VB, Romano SJ, Vecchi GM. Role Playing: applications in hostage and crisis negotiation skills training. Behav Modif 2008;32(2):248-63.

39. Kottow M. Literary narrative in medical practice. Med Humanities. 2002;28(1):41-4.

40. Zinker J. Processo criativo em Gestalt-terapia. São Paulo: Summus; 2007. p.223.

41. Thom DH. Training physicians to increase patient trust. J Eval Clin Pract. 2000;14(6):245-53.

42. Resnick M, Wilensky U. Diving into complexity: developing probabilistic decentralized thinking through role-playing activities. J Learning Science. 1998;7(2):153-72.

43. Bellack AS et al. Role play for assessing the social competence of psychiatric patients. Psychol Assess. 1990;2(3):248-55.

44. Nikendei C, Zeuch A, Dieckmann P, Roth C, Schäfer S, Völkl $\mathrm{M}$ et al. Role-playing for more realistic technical skills training. Med Teach. 2005;27(2):122-6.

\section{CONTRIBUIÇÃO DOS AUTORES}

Natália B. H. Jucá, autora principal, tutora da coleta de dados, ministrante das aulas e das atividades de base ao artigo; definição e discussão do tema; autora da introdução, objetivos, metodologia; organização, estruturação de revisão bibliográfica e revisão geral do artigo.

Annatália M. de Amorim Gomes, orientadora, ministrante das aulas e das atividades de base ao artigo, reestruturação do título, introdução e objetivos, metodologia, organização, revisão geral do artigo. 
Lucas S. Mendes, Diego M. Gomes, Bruna Vitória L. Martins contribuiu na coleta de dados, revisão bibliográfica/ fichamento e da redação do artigo.

Carlos Maximiliano Gaspar Carvalho Heil Silva, Carolina Arcanjo Lino e Kathiane Lustosa Augusto, ministrantes das aulas e das atividades de base ao artigo, paticiparam da redação do artigo. Andrea Caprara, coorientador, ministrante das aulas e das atividades de base ao artigo; responsável pela organização dos dados, revisão, reestruturação de título e revisão bibliográfica.

\section{CONFLITO DE INTERESSES}

Declarou não haver.

\section{ENDEREÇO PARA CORRESPONDÊNCIA}

Natália Braga Hortêncio Jucá

Centro de Ciências da Saúde

Universidade Estadual do Ceará.

Av. Padre Antônio Tomás, 150 - apto. 701

Aldeota - Fortaleza

CEP. 60140-160 CE

E-mail: nataliabraga@superig.com.br 